\title{
Etude en laboratoire d'un amortisseur de houle constitué d'une plage parabolique et de deux plaques verticales perforées.
}

\author{
Maria Cristina GARCIA GOVEA ${ }^{1}$, Daniel LEVACHER ${ }^{2}$, Daniel \\ CAMINADE $^{3}$ Christophe CHEVALIER $^{4}$
}

${ }^{1}$ Doctorante, ${ }^{2}$ Professeur, ${ }^{4}$ Docteur, Université de Caen, Morphodynamique Continentale et Côtière, 24, rue des Tilleuls, 14 o00 Caen, Tel 02315657 09, Fax 0231565757

${ }^{3}$ Maître de Conférences, ISA-BTP, Laboratoire des Sciences Appliquées au Génie Civil (LASAGEC), 1, allée du Parc Montaury Anglet.

\section{RESUME}

Ce travail présente l'étude d'une structure d'amortissement de la houle. L'amortisseur est constitué d'une structure métallique parabolique et de deux plaques verticales. La structure comme les plaques sont perforées. La porosité de la parabole est de $48 \%$, elle est de $40 \%$ pour la première plaque verticale et de $30 \%$ pour la deuxième plaque.

Des essais ont été menés en canal à houle, pour une profondeur de 0,50 mètre où plusieurs périodes et hauteurs de houle incidente ont été simulées. Pour obtenir une amélioration de l'efficacité de l'amortisseur, la porosité de la structure a été modifiée en utilisant un filtre à air de type média filtrant.

Cette étude a été réalisée dans un des canaux à houle du Centre de Géomorphologic de l'Université de Caen.

\begin{abstract}
The aim of this work is an experimental study of a wave absorber. The absorber combines a parabolic porous structure and two vertical perforated plates behind the parabola. All the elements are made with steel sheet. The porosity of each element is respectively $48 \%$ for the parabola, $40 \%$ for the first plate and $30 \%$ for the second plate.
\end{abstract}

The absorber was tested for a water depth of 0,50 meter and for a wide range of wave periods and wave heights. In order to make more efficient the absorber, a porous filter has been added on the surface profile.

This study has been conducted in on of the flumes of the Centre de Géomorphologie of the University of Caen 


\section{Introduction}

Un des facteurs qui modifie la houle générée par le batteur lors d'essais en laboratoire est la réflexion des vagues incidentes sur le fond du canal à houle. Les maquettes en laboratoire sont en général de dimensions réduites et il est indispensable de disposer à l'extrémité du canal à houle des conditions aux limites aussi absorbantes que possible. Afin d'éviter les réflexions multiples qui viennent perturber la zone d'essais, un amortisseur de houle a été mis au point et testé.

\section{Description du dispositif expérimental}

\subsection{Equipements généraux}

Des essais en laboratoire ont été entrepris dans le canal à houle du Centre de Géomorphologie de l'Université de Caen, dont les principales caractéristiques géométriques sont les suivantes:

$$
\begin{aligned}
& \text { Longueur utile: } \quad 22,00 \mathrm{~m} \\
& \text { Largeur : } \quad 0,80 \mathrm{~m} \\
& \text { Hauteur utile : } \quad 0,80 \mathrm{~m}
\end{aligned}
$$

Le canal était initialement équipé d'un générateur de houle régulière, désormais il est doté d'un générateur de houle aléatoire. Le générateur avec lequel ont été réalisés les essais est un générateur de type batteur plan. Son mouvement est obtenu par un moteur à courant continu permettant de générer une houle régulière dont la période est réglée par un variateur de courant. La variation de l'amplitude du mouvement de la pale qui conditionnera l'amplitude de l'onde est obtenue par réglage d'un plateau excentrique monté en bout d'arbre sur un motoréducteur.

Pour le dépouillement de la houle nous avons appliqué la méthode de Goda [3]en utilisant trois sondes séparées par des distances différentes entre elles [4].

\subsection{L'amortisseur de houle}

L'amortisseur de houle a été disposé à l'extrémité du canal face au générateur de houle. Le but de l'amortisseur de houle est de réduire au minimum les réflexions de la houle. L'amortisseur (figure 1) est composé d'une plage parabolique poreuse en acier galvanisé dont les caractéristiques géométriques sont les suivantes:

$\begin{array}{lll}\text { Hauteur } & : & 0,60 \mathrm{~m} \\ \text { Largeur } & : & 0,79 \mathrm{~m} \\ \text { Longueur } & : & 1,30 \mathrm{~m}\end{array}$


Epaisseur : $\quad 1,50 \mathrm{~mm}$

Equation de la parabole : $y^{2}=4 a x$, avec $a=6,923$ et $x$ et $y$ en $\mathrm{cm}$.

et par deux plaques verticales poreuses en acier galvanisé dont les caractéristiques géométriques sont :

$\begin{array}{lll}\text { Hauteur } & : & 0,80 \mathrm{~m} \\ \text { Largeur } & : & 0,79 \mathrm{~m} \\ \text { Epaisseur } & : & 1,50 \mathrm{~mm}\end{array}$

Les trous de la parabole et des plaques verticales sont de section circulaire. La porosité est définie comme le rapport de la surface des orifices à la surface totale de la plaque. La porosité des plaques décroît au fur et à mesure que la distance au générateur de houle augmente. La houle rencontre ainsi au cours de sa propagation des plaques de moins en moins perméables. Ainsi, la porosité de la parabole est de $48 \%$, la porosité de la plaque $n^{\circ} 1$ est de $40 \%$ et de la plaque $n^{\circ} 2$ est de $30 \%$.

\section{PLAGE PARABOLIQUE}

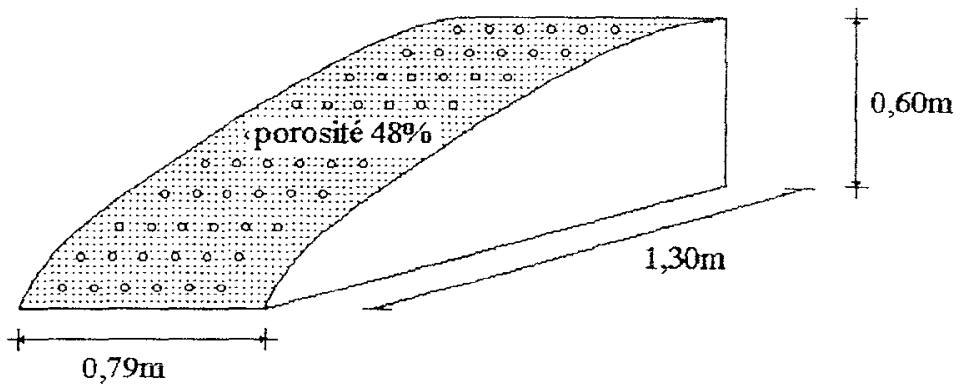

PLAQUES VERTICALES

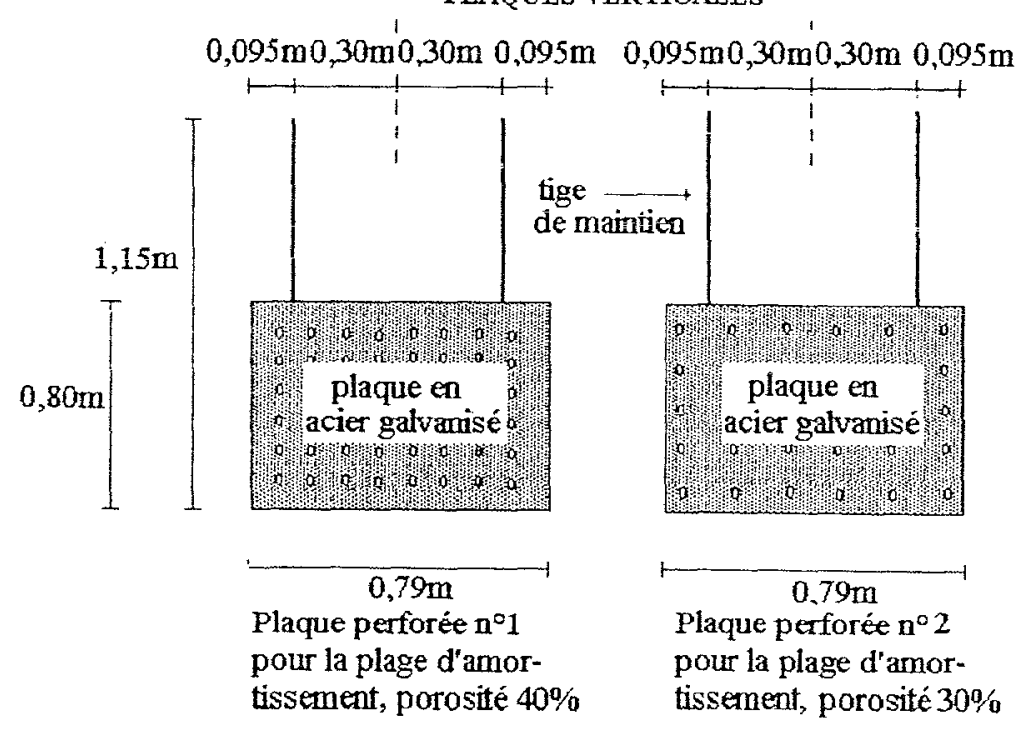

Figure 1. Configuration de la structure d'amortissement. Wave absorber configuration. 
L'espacement entre la parabole et les plaques verticales varie selon la série d'essais effectuée (figure 2), à savoir : $X=0,50 \mathrm{~m}$ ou $0,75 \mathrm{~m}, Y=0,50 \mathrm{~m}$ ou $0,75 \mathrm{~m}$, $Z=0,60 \mathrm{~m}$ ou $0,85 \mathrm{~m}$. De plus, pour chaque essai, on a mis des filtres à air sur la parabole et les plaques verticales.

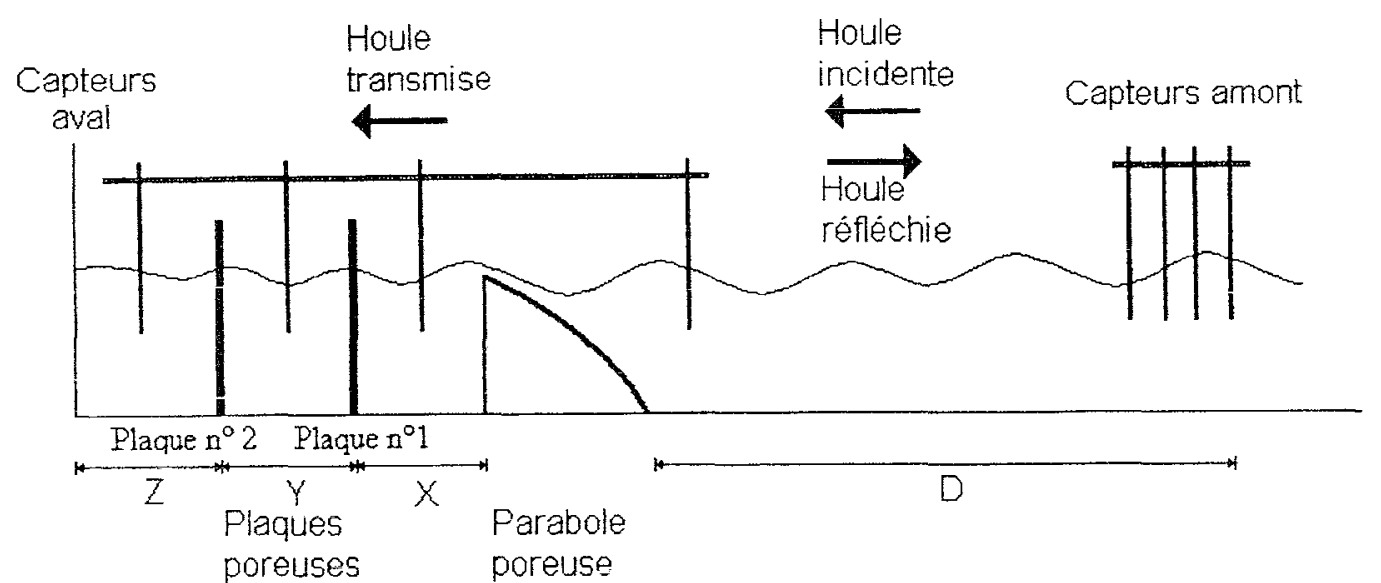

Figure 2. Disposition des plaques et capteurs de mesure de la houle. Experimental seiup.

\section{Les conditions hydrodynamiques des essais}

Les essais en laboratoire comportent quatre séries d'essais, la hauteur d'eau dans le canal a été fixée à 0,50 mètre.

La première série d'essais a été effectuée sans aucun filtre disposé sur la structure d'amortissement. L'étendue des périodes (T) explorées va de 1 s à 2,5s. Pour chaque période, les mesures ont été menées avec 6 amplitudes de houle soit 6 excentricités du batteur différentes, jusqu'aux limites de course du batteur. $L$ 'intervalle inter-plaques est de $X=0,50 \mathrm{~m}, Y=0,50 \mathrm{~m}$ et $\mathrm{Z}=0,60 \mathrm{~m}$.

La deuxième série d'essais a été réalisée avec un filtre destiné à filtrer l'air dont les caractéristiques sont décrites ci-dessous. Ces filtres ont été installés sur la parabole et les plaques verticales. Neuf périodes ont été étudiées : $\mathrm{T}=1 \mathrm{~s}, \mathrm{~T}=1,15 \mathrm{~s}$, $T=1,25 \mathrm{~s}, T=1,30 \mathrm{~s}, \mathrm{~T}=1,50 \mathrm{~s}, \mathrm{~T}=1,80 \mathrm{~s}, \mathrm{~T}=2 \mathrm{~s}, \mathrm{~T}=2,20 \mathrm{~s}$, et $\mathrm{T}=2,50 \mathrm{~s}$. Pour chaque période, les mesures ont été faites avec 6 amplitudes de houle. Les intervalles entre l'arrière de la parabole, les plaques et le fond du canal sont identiques à ceux de la première série d'essais. Le filtre à air utilisé de référence média filtrant de type RCP100 est en fibres synthétiques de $5 \mathrm{~mm}$ d'épaisseur. Il est employé dans diverses applications de pré-filtration pour le traitement de l'air dans la ventilation et le conditionnement pour retenir les particules supérieures à $1 \mu \mathrm{m}$. Il a une efficacité de $97 \%$ ashare gravimétrique, un classement au feu $\mathrm{M} 1$, et sa classification est EU4/G4.

En ce qui concerne la troisième série d'essais, la demière plaque verticale de l'amortisseur a été supprimée dans le but de vérifier son efficacité. Le filtre à air 
est resté installé sur la parabole et la plaque verticale restante. La séparation entre la parabole et la plaque a été de $X=0,50 \mathrm{~m}$ et $\mathrm{Y}=1,10 \mathrm{~m}$. Deux périodes ont été étudiées $\mathrm{T}=1,50 \mathrm{~s}$ et $\mathrm{T}=1,25 \mathrm{~s}$ et pour chaque période les mesures ont été aussi effectuées avec 6 amplitudes de houle.

Pour la quatrième série d'essais, la position de la parabole a été modifiée comme celles des plaques, soit $X=0,75 \mathrm{~m}, Y=0,75 \mathrm{~m}$ et $\mathrm{Z}=0,85 \mathrm{~m}$. La gamme des périodes explorées va de $T=1,50 \mathrm{~s}$ à $T=2 \mathrm{~s}$. Quatre périodes ont été étudiées : $1,50 \mathrm{~s}$, $1,60 \mathrm{~s}, 1,80 \mathrm{~s}$ et $2 \mathrm{~s}$. Pour chaque période les mesures ont toujours été effectuées avec 6 amplitudes de houle.

Les valeurs concernant la longueur d'onde $L$, la profondeur relative $d / L$ (ou $\mathrm{kd}=2 \pi \mathrm{d} / \mathrm{L}$, étant $\mathrm{k}$ le nombre d'onde) sont données dans le tableau suivant:

\begin{tabular}{|c|c|c|c|c|c|c|c|c|c|c|}
\hline $\mathrm{T}(\mathrm{s})$ & 1 & 1,15 & 1,25 & 1,30 & 1,50 & 1,60 & 1,80 & 2,20 & 2,20 & 2,50 \\
\hline $\mathrm{L}(\mathrm{m})$ & 1,51 & 1,92 & 2,18 & 2,31 & 2,83 & 3,08 & 3,57 & 4,05 & 4,53 & 5,23 \\
\hline $\mathrm{d} / \mathrm{L}$ & 0,33 & 0,26 & 0,23 & 0,22 & 0,18 & 0,16 & 0,14 & 0,12 & 0,11 & 0,10 \\
\hline $\mathrm{kd}$ & 2,08 & 1,64 & 1,44 & 1,35 & 1,11 & 1,02 & 0,88 & 0,77 & 0,69 & 0,60 \\
\hline
\end{tabular}

Tableau 1. Principales valeurs caractéristiques de houle pour $\mathrm{d}=0,50 \mathrm{~m}$.

Characteristic wave values for $d=0,50 \mathrm{~m}$.

\section{Paramètres d'en registrement de la houle}

De façon à éviter les phénomènes perturbateurs dus aux réflexions multiples sur l'amortisseur et le batteur de houle, les acquisitions sont faites avant que la houle une première fois réfléchie par l'amortisseur, puis par le batteur ne parvienne jusqu'aux capteurs de houle [1]. Si l'on se réfêre à la figure 3 qui illustre le trajet de la houle après le démarrage du batteur $\left(T_{0}=0\right)$ le temps d'attente minimal avant de déclencher l'acquisition est:

$$
\mathrm{T} 1=(\mathrm{d} 1+\mathrm{d} 2) / \mathrm{Cg}
$$

où Cg est la vitesse de groupe de la houle (vitesse de propagation de l'énergie). Le temps final d'acquisition doit être inférieur à

$$
\mathrm{T} 2=\mathrm{T} 1+(2 \mathrm{~d} 3 / \mathrm{Cg})
$$




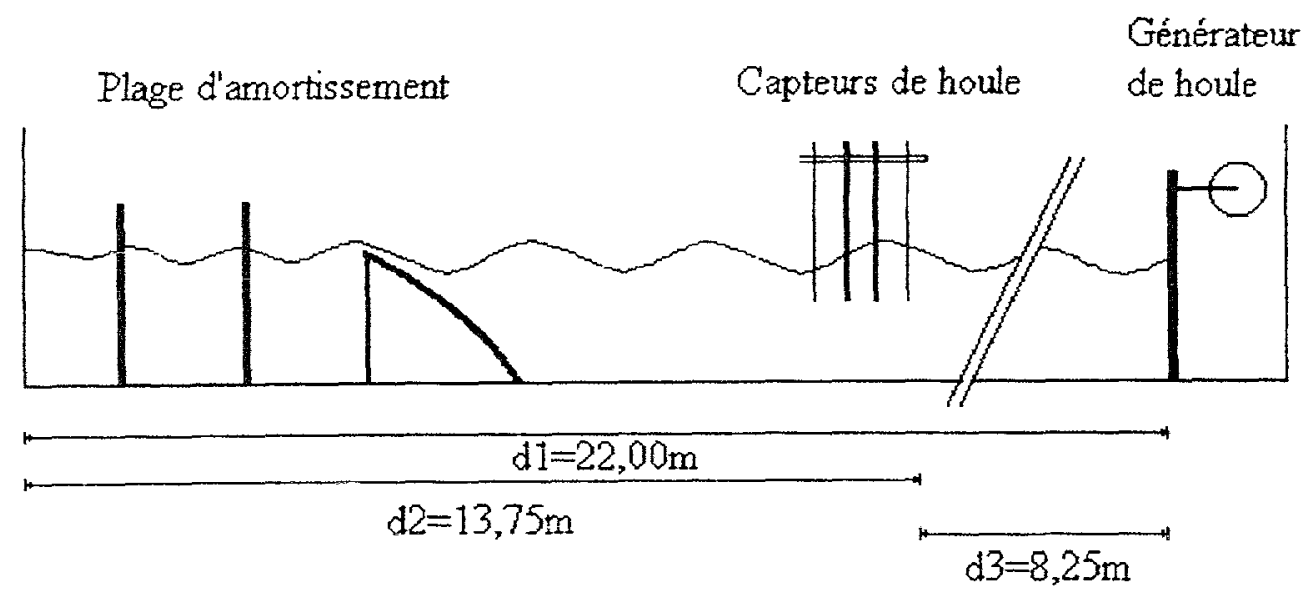

Figure 3. Trajets parcourus par la houle. Distances ran by the waves.

Les caractéristiques d'acquisition récapitulées sont regroupées dans le tableau 2.

\begin{tabular}{|c|c|c|c|c|c|c|}
\hline $\begin{array}{c}\text { Période } \\
\text { de houle } \\
\mathrm{T}(\mathrm{s})\end{array}$ & $\begin{array}{c}\text { Vitesse } \\
\text { de groupe } \\
\mathrm{Cg}(\mathrm{m} / \mathrm{s})\end{array}$ & $\begin{array}{c}\mathrm{T} 1 \\
\text { théorique } \\
(\mathrm{s})\end{array}$ & $\begin{array}{c}\mathrm{T} 2 \\
\text { théorique } \\
(\mathrm{s})\end{array}$ & $\begin{array}{c}\text { Date } \\
\text { Début } \\
(\mathrm{s})\end{array}$ & $\begin{array}{c}\text { Durée } \\
\text { Acquisition } \\
(\mathrm{s})\end{array}$ & $\begin{array}{c}\text { Nombre } \\
\text { de } \\
\text { vagues }\end{array}$ \\
\hline 1,00 & 0,85 & 42 & 61 & 42 & 19 & 19 \\
\hline 1,15 & 1,04 & 34 & 50 & 34 & 16 & 13 \\
\hline 1,25 & 1,16 & 31 & 45 & 31 & 14 & 11 \\
\hline 1,30 & 1,21 & 30 & 43 & 30 & 13 & 10 \\
\hline 1,50 & 1,40 & 25 & 37 & 25 & 12 & 8 \\
\hline 1,60 & 1,35 & 26 & 39 & 26 & 13 & 8 \\
\hline 1,80 & 1,61 & 22 & 32 & 22 & 10 & 5 \\
\hline 2,00 & 1,71 & 21 & 31 & 21 & 10 & 5 \\
\hline 2,20 & 1,79 & 20 & 29 & 20 & 9 & 4 \\
\hline 2,50 & 1,88 & 19 & 28 & 19 & 9 & 3 \\
\hline
\end{tabular}

Tableau 2. Principales caractéristiques d'acquisition pour $\mathrm{d}=0,50 \mathrm{~m}$. Acquisition values parameters for $d=0,50 \mathrm{~m}$.

\section{Résultats}

\subsection{Structure d'amortissement sans filtre}

Les résultats obtenus sont assez médiocres car la parabole est trop perméable, donc la dissipation de l'énergie reste faible. Les coefficients de réflexion sont de l'ordre de 68 à $20 \%$ pour les cambrures les plus faibles inférieures à $2 \%$. Avec des cambrures supérieures à $2 \%$ on obtient des coefficients de réflexion de l'ordre de $20 \%$ avec un minimum à $4 \%$ (figure 4 ). 


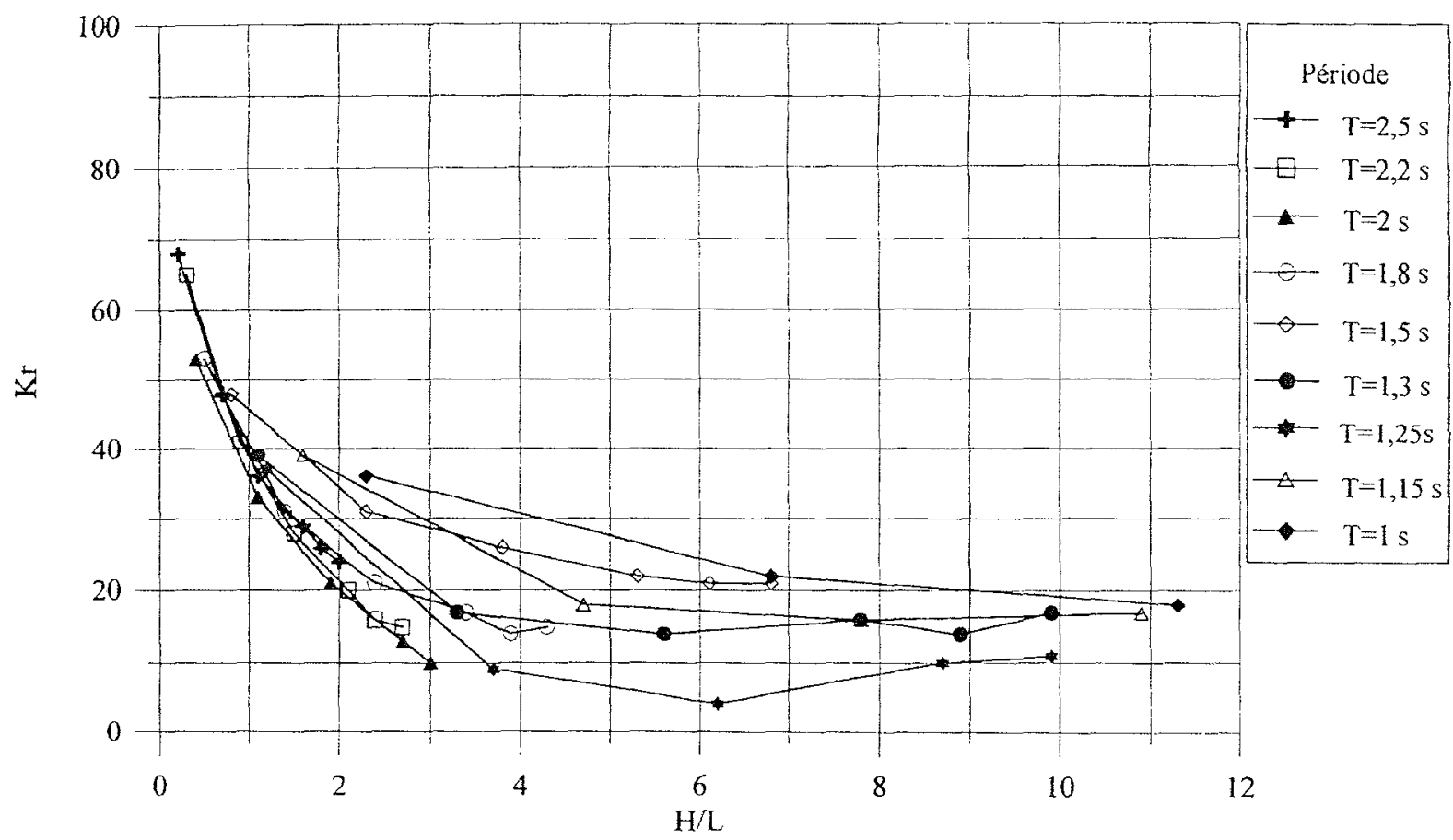

Figure 4. Evolution du coefficient de réflexion $\mathrm{Kr}$ en fonction de la cambrure. Cas de la structure d'amortissement sans filtre. Reflection coefficient versus wave steepness for a wave absorber without filter.

\subsection{Structure d'amortissement avec filtre}

Les résultats précédents nous ont conduit à changer la porosité de la structure en utilisant un filtre de type media filtrant. Les résultats font l'objet des graphiques de la figure 5. Une notable amélioration des résultats est obtenue pour cette configuration de la structure. Les coefficients de réflexion varient entre 25 et $3 \%$, résultats tout à fait corrects pour toùte la gamme de périodes testées. Le comportement de l'amortisseur est optimal pour les périodes les plus courtes $T=1,00 \mathrm{~s}$ et $\mathrm{T}=1,15 \mathrm{~s}$ où les coefficients de réflexion atteignent seulement des valeurs de 3 à $11 \%$.

Cette diminution des valeurs du coefficient de réflexion est due principalement à la dissipation de l'énergie provoquée par un déferlement sur la parabole.

\subsection{Influence de la dernière plaque verticale}

Pour vérifier l'efficacité de la dernière plaque verticale nous l'avons supprimée de l'ensemble de l'amortisseur. On a choisi les périodes $T=1,50 \mathrm{~s}$ et $T=1,25 \mathrm{~s}$ comme représentatives pour tester cette configuration. Les résultats obtenus (figure 6) montrent que le comportement du système n'est pas fondamentalement modifié. Les valeurs du coefficient de réflexion restent presque constantes par rapport au cas précédent. 


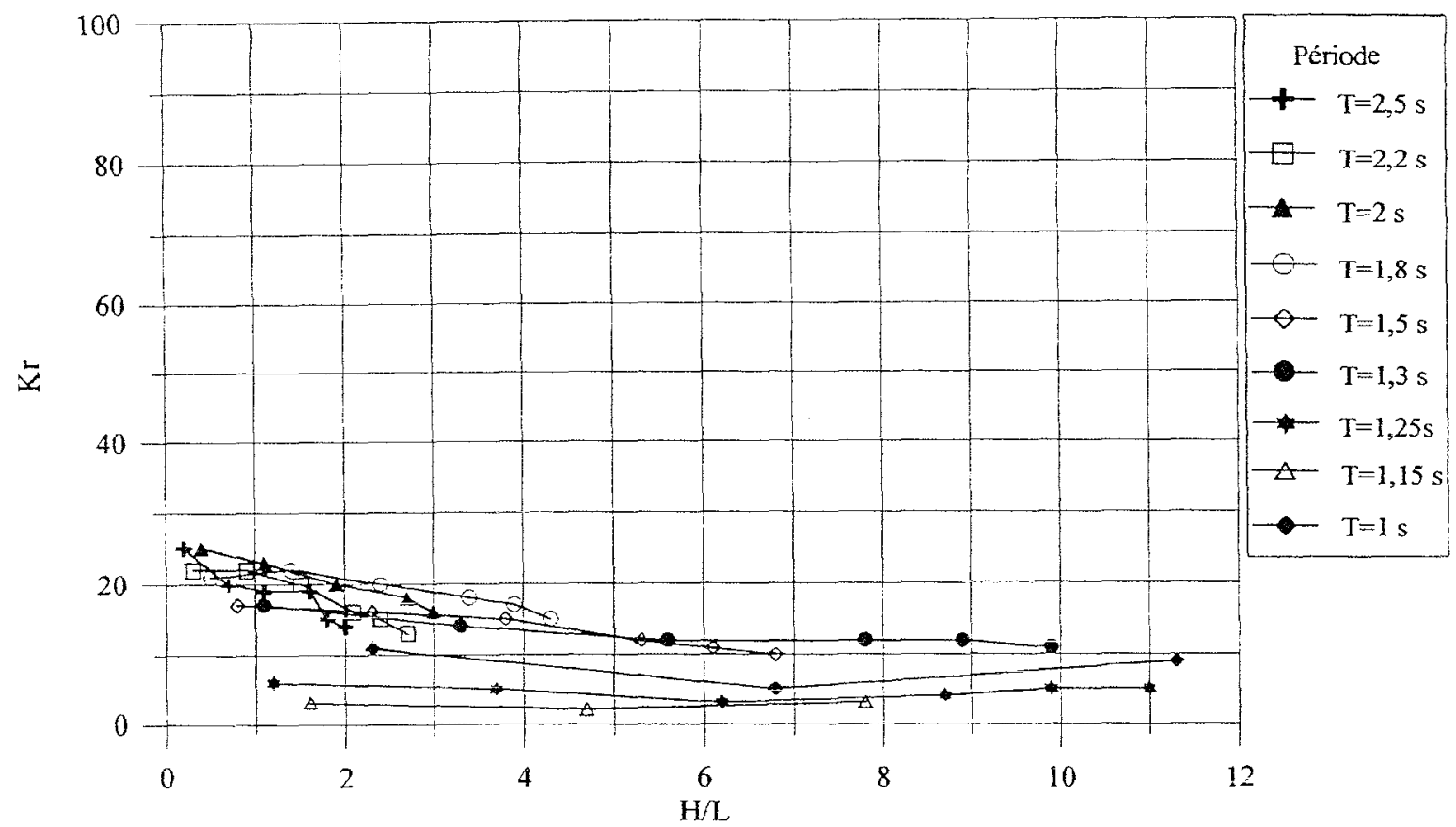

Figure 5. Evolution du coefficient de réflexion $\mathrm{Kr}$ en fonction de la cambrure. Cas de la structure d'amortissement avec filtre. Reflection coefficient versus wove steepness for a wave absorber with filter.

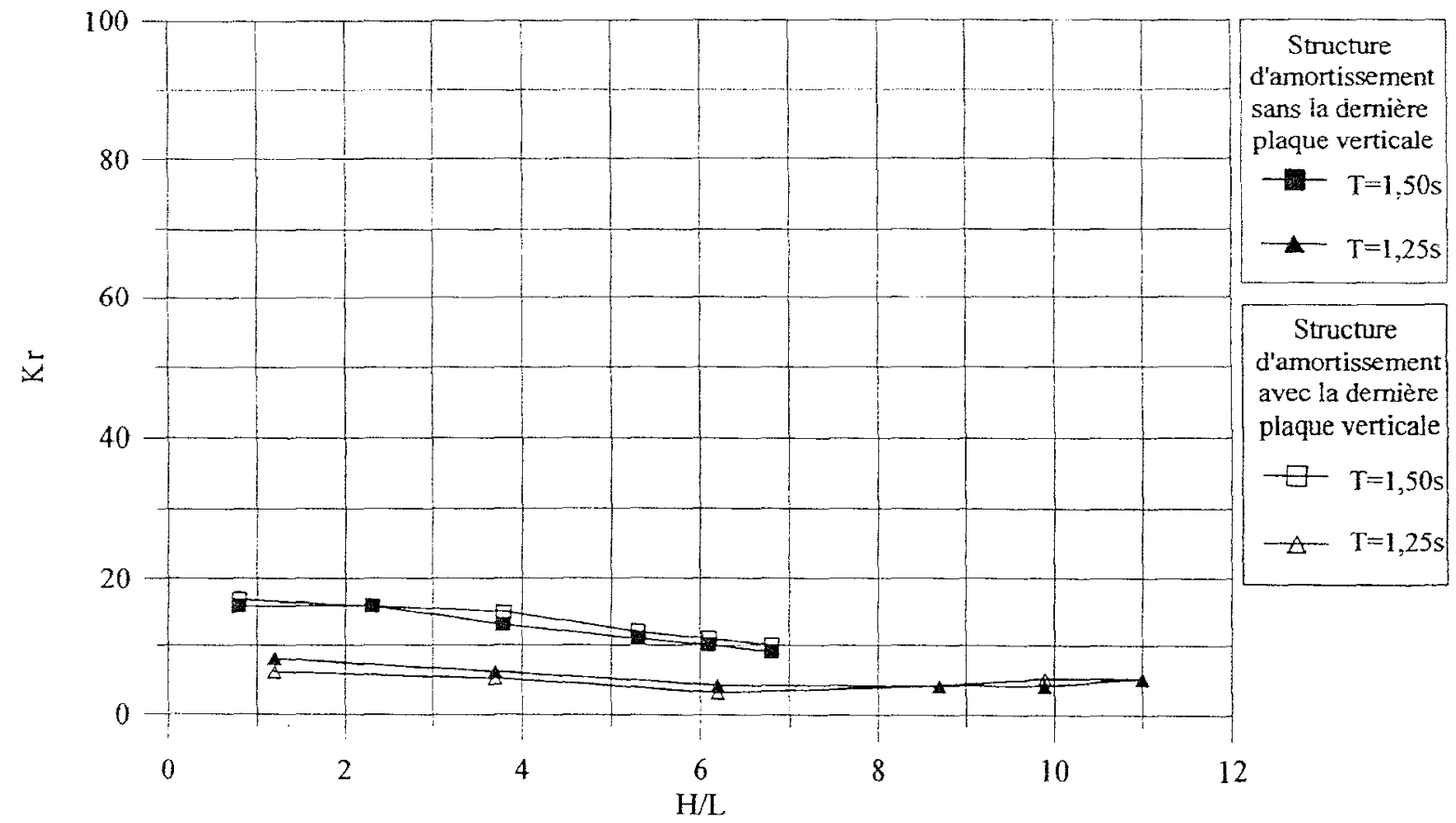

Figure 6. Evolution du coefficient de réflexion $\mathrm{Kr}$ en fonction de la cambrure: influence de la dernière plaque verticale. Reflection coefficient versus wave steepness: influence of the last plate. 


\subsection{Influence de l'écartement entre la parabole et les plaques verticales}

Pour tester l'influence de l'écartement entre la parabole et les plaques, les distances ont été modifiées de $X=0,50 \mathrm{~m}, Y=0,50 \mathrm{~m}$ et $Z=0,60 \mathrm{~m}$ (conditions initiales) à $X=0,75 \mathrm{~m}, Y=0,75 \mathrm{~m}$ et $\mathrm{Z}=0,85 \mathrm{~m}$.

Des cas précédents étudiés, on a observé que pour les périodes $T=1,15 \mathrm{~s}$ et $\mathrm{T}=1,25 \mathrm{~s}$, les coefficients de réflexion sont relativement stables à de faibles valeurs : moins de $3 \%$ pour la période $\mathrm{T}=1,15 \mathrm{~s}$ et moins de $6 \%$ pour la période $\mathrm{T}=1,25 \mathrm{~s}$. Ceci semble indiquer qu'on se situe dans la plage des conditions où le fonctionnement de l'amortisseur est optimal. Pour ces deux périodes, la longueur de séparation entre la parabole et les plaques verticales est de l'ordre du quart de la longueur de l'onde.

$$
\begin{array}{lll}
\mathrm{T}=1,15 \mathrm{~s} & \mathrm{~L}=1,92 \mathrm{~m} & 1 / 4=0,48 \mathrm{~m} \\
\mathrm{~T}=1,25 \mathrm{~s} & \mathrm{~L}=2,18 \mathrm{~m} & 1 / 4=0,55 \mathrm{~m}
\end{array}
$$

Pour confirmer cette observation les périodes $T=1,50 \mathrm{~s}, \mathrm{~T}=1,60 \mathrm{~s}, \mathrm{~T}=1,80 \mathrm{~s}$ et $\mathrm{T}=2,00 \mathrm{~s}$ ont été choisies. Les résultats obtenus (figure7) montrent que l'amortisseur ne présente aucune amélioration sensible, les valeurs du coefficient de réflexion restent assez élevées. Seulement pour la période $T=1,50 \mathrm{~s}$ et pour certaines cambrures, une légère diminution de $\mathrm{Kr}$ a été notée.

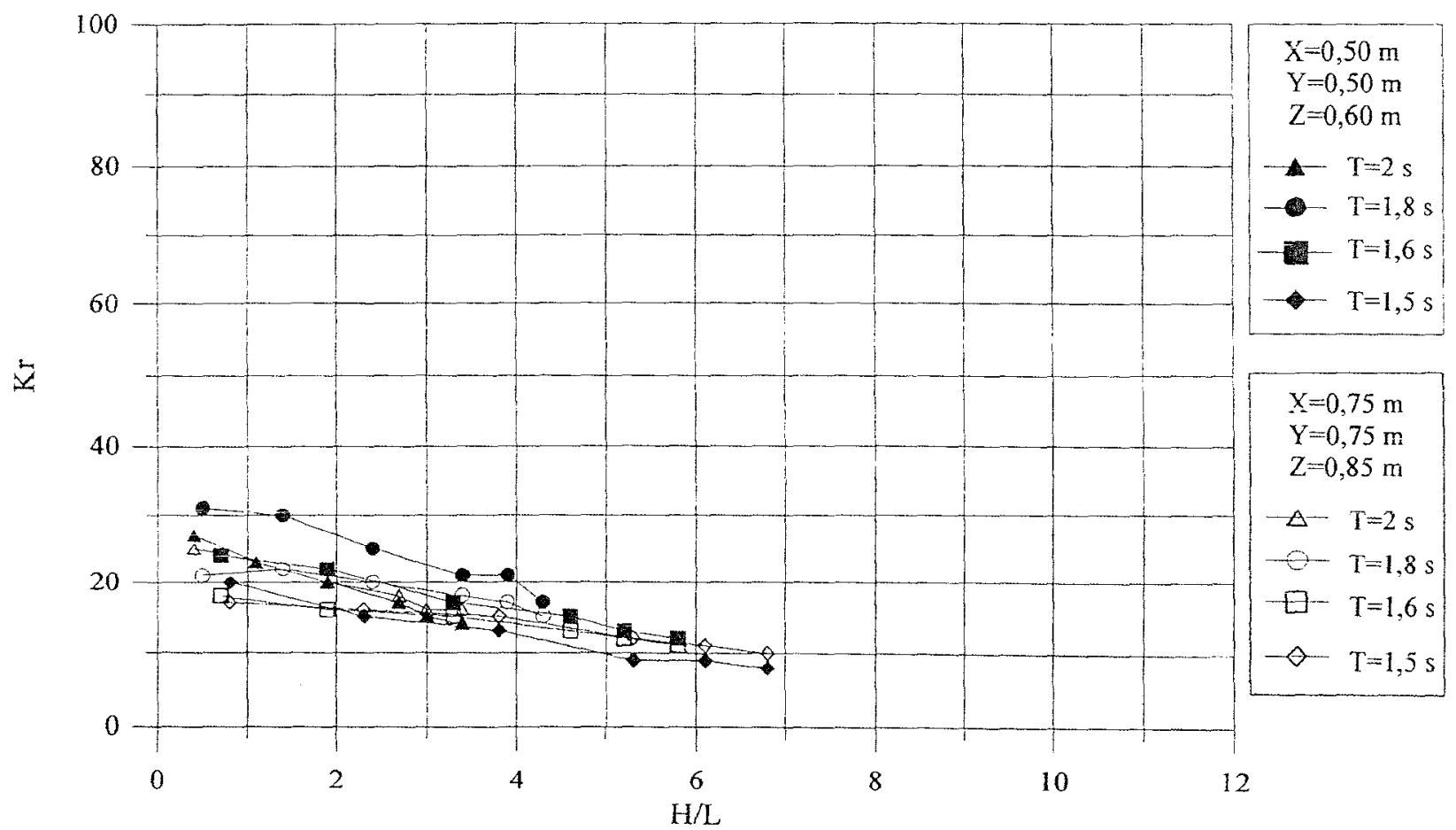

Figure 7. Evolution du coefficient de réflexion $\mathrm{Kr}$ en fonction de la cambrure: influence de l'écartement entre la parabole et les plaques verticales. Reflection coefficient versus wave steepness: influence of the distances between the parabola and the plates. 


\section{Conclusion}

Les essais qui ont été réalisés en laboratoire ont permis de caractériser le comportement de l'amortisseur de houle composé d'une structure parabolique et de deux plaques verticales. Il nous ont permis de réaliser les observations suivantes:

-L'amortisseur de houle avec un filtre possède une efficacité optimale pour les périodes $T=1,00 \mathrm{~s}, T=1,15 \mathrm{~s}$ et $\mathrm{T}=1,25 \mathrm{~s}$ pour lesquelles le coefficient de réflexion ne dépasse pas $10 \%$, pour le reste des périodes l'amortisseur reste assez efficace avec des valeurs de $\mathrm{Kr}$ ne dépassant pas $25 \%$.

-Le fait de supprimer la demière plaque verticale de l'ensemble de l'amortisseur ainsi que de changer l'écartement inter-plaques n'apportent aucune amélioration.

A partir de ces ob́servations il apparaît que pour les faibles cambrures, il faut travailler dans la masse du fluide. Dans le cas des grandes cambrures pour que le déferlement soit alors efficace il faut régler la parabole en fonction de la hauteur d'eau.

Le système étudié répond partiellement aux exigences des plages d'amortissement pour lesquelles on attend des coefficients de réflexion ne dépassant pas $10 \%$, soit une efficacité de $99 \%$ en termes d'énergie.

\section{Réferences}

[1] ALLILAIRE J., BENOIT M. Essais en laboratoire d'un amortisseur de houle multi-écrans à plaques verticales perforées. Rapport technique No HE42/94/038/A, Direction des Etudes et Recherches, Laboratoire National d'Hydraulique, Electricité de France, (1984), 28p.

[2] GARCIA-GOVEA C., LEVACHER D., CAMINADE D. Etude en laboratoire d'un amortisseur de houle constitué d'une plage parabolique et de deux plaques verticales perforées. Rapport technique, Centre de Géomorphologie, Université de Caen, (1999), 57p.

[3] HUGHES S. Physical models and laboratory techniques in coastal engineering. Advanced Series on Ocean Engineering, volume 7, World Scientific, Coastal Engineering Research Center, Waterways Experimental Station, USA (1993), $560 \mathrm{p}$.

[4] MANSARD E., FUNKE E. The measurement of incident and reflected spectra using a least square method. Proc. 17 th Coastal Engineering Conference, Sydney, Australia, (1980), pp. 154-172.

[5] SARPKAYA T., ISAACSON M. Mechanics of wave forces on offshore structures. Van Nostrand, Reinhold Company (1981), 651 p. 